\title{
Solitary bone plasmacytoma compression injury disguised as back pain: a case report
}

\author{
Steven D. Jackson ${ }^{1,2} \cdot$ Bethany A. Wiering ${ }^{1,3} \cdot$ Amanda A. Herrmann ${ }^{1,3} \cdot$ MacKenna A. Hinz ${ }^{1}$ Leah R. Hanson ${ }^{1,3}$
}

Received: 2 October 2018 / Revised: 21 January 2019 / Accepted: 27 January 2019

(c) International Spinal Cord Society 2019

\begin{abstract}
Introduction Low back pain is a leading disability worldwide; however, it is not often the result of a serious underlying condition such as a tumor. As a result, diagnosis of a serious underlying cause of low back pain may be delayed, such as in this case.

Case presentation We describe a case of a man presenting with low back pain, who was eventually diagnosed with solitary bone plasmacytoma (SBP) causing spinal cord compression from approximately T7-T9. The patient was classified as T8 ASIA C-Incomplete Paraplegia. He underwent an emergency T7-T9 open posterior laminectomy and resection of the epidural mass/tumor. Following an intensive course of rehabilitation treatment, the patient progressed to ASIA D.

Discussion Although SBP of the spine is rare, back or neck pain is a common initial presentation. This case is unique in that we provide a detailed description of both medical and rehabilitation diagnosis and treatment. We also suggest that persistent back pain warrants complete MRI spinal imaging to provide proper diagnosis and prompt treatment for cases with a serious underlying condition.
\end{abstract}

\section{Introduction}

Low back pain has been reported as the leading cause of disability worldwide [1]. It is frequently mechanical in nature (e.g., spondylosis) and is not typically the result of a serious underlying cause (e.g., tumors) [2]. Consequently, diagnosis of uncommon and life-threatening causes of back pain may often be delayed, such as in this case. This case report discusses a patient who initially presented with low back pain, which eventually was determined to be caused by a solitary bone plasmacytoma (SBP). Solitary plasmacytoma (SP) is a rare type of plasma cell dyscrasia [3]. In this case, an even rarer phenomenon is presented: spinal cord compression due to plasma cell infiltration. This case report describes a patient who suffered a SCI due to SBP, which was initially believed to be unspecified low back pain. This report provides a comprehensive description of

\footnotetext{
Steven D. Jackson

Steven.D.Jackson@HealthPartners.com

HealthPartners Neuroscience Center, St. Paul, MN, USA

2 Regions Hospital, St. Paul, MN, USA

3 HealthPartners Institute, Minneapolis, MN, USA
}

the radiographic assessment, medical and surgical management, and rehabilitation treatment, which lead to a full functional recovery.

\section{Case presentation}

A 67 year old African American male with a history of diabetes mellitus type II, hypertension, and hypercholesterolemia presented to the emergency department (ED) in October 2016. He was evaluated for a constant nonpalpable low back (left > right) "pressure" and atraumatic pain, with discomfort for about $8 \mathrm{~h}$. He was treated with ibuprofen and experienced pain improvement. A urine analysis test was clear and a chest X-ray was unremarkable, therefore he was discharged home. He was diagnosed with back pain with an unspecified location, laterality, and chronicity.

The patient was asymptomatic until he presented for a primary care clinic visit in February 2017 with left flank pain, which began a week prior. He stated the non-radiating pain was occurring daily and was worse at night. He alleviated symptoms with $800 \mathrm{mg}$ ibuprofen. From 2/2017-4/ 2017 , he was seen by a primary care physician during 4 office visits, spoke to a careline nurse twice, and was seen by a chiropractor for 3 visits. An ultrasound showed a 

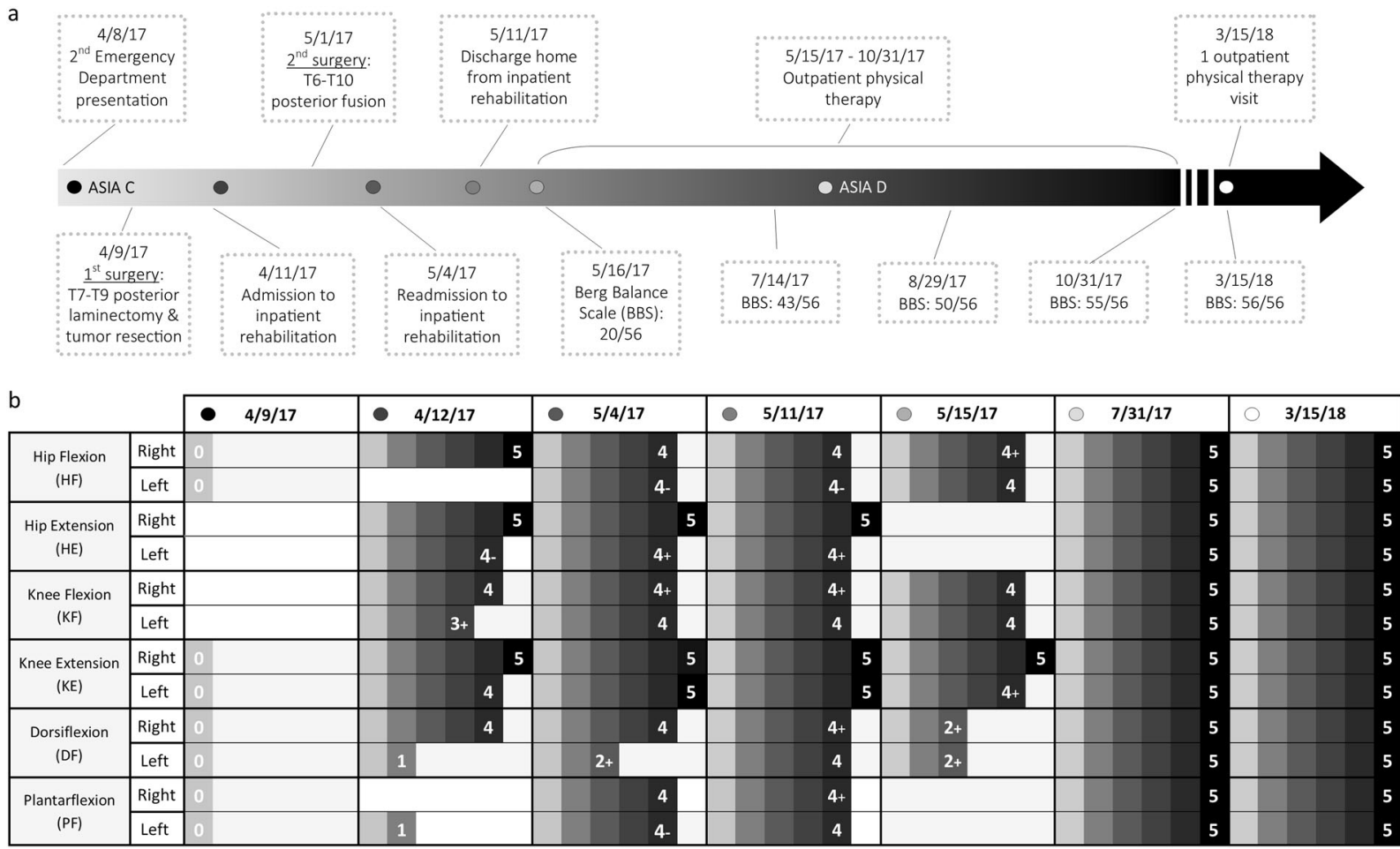

Fig. 1 Timeline of progression to ASIA D and manual muscle testing (MMT) scores. a Timeline of patient's progression from ASIA A to ASIA D. b Progression of patient's right and left lower extremity

possible small kidney stone, however the pancreas, metabolic panel, and urine analysis were all normal. He was prescribed hydrocodone/acetaminophen, diclofenac sodium, and a muscle relaxant to alleviate symptoms. A lumbar Xray showed degenerative joint disease, retrolisthesis from L2-3 to L4-L5, and a partial collapse of L5. A MRI of his lumbar spine showed degenerative joint disease and spondylosis. He received 3 sessions of chiropractic care and planned to follow up with a Physical Medicine and Rehabilitation (PM\&R) physician and begin physical therapy (PT).

Prior to his PM\&R and PT appointments, he presented again to the ED in April 2017 for evaluation of sudden onset right inferoscapular pain (Fig. 1a). He described the pain as 10/10, a "burning, intense, localized" sensation without radiation. At this time, he was managing chronic back pain with the following medications: tizanidine, gabapentin, and diclofenac sodium; however, he expressed that the current pain was different than his typical pain. Based on the ED evaluation, he had no leg pain or swelling, however he stated that the back pain was worse when he moved his arms. He denied neck pain and presented no numbness, tingling, or headache. In addition, he had no bowel or bladder symptoms. He was admitted for observation and to rule out acute coronary syndrome. manual muscle testing scores over time. MMT scores range from $0-5$, with 0 indicating no palpable or observable muscle contraction and 5 indicating normal function

Overnight, he had increased back pain with acute numbness and tingling in his lower extremities. He was unable to void and noted weakness and a sensory deficit across his abdomen. In the morning, nursing performed a post-void residual urine test, which was $725 \mathrm{~mL}$. He did not have the desire to urinate, even with the large amount of urine in his bladder. By mid-day, he was unable to move his lower extremities and his sensation was diminished below T6. A manual muscle test (MMT) revealed no muscle contractions in his bilateral lower extremities (BLE; Fig. 1b). He presented with no deficits or symptoms in the upper extremities, face, or head.

The patient underwent MRI of the thoracic spine, which revealed a $5 \times 1.2 \mathrm{~cm}$ epidural mass causing spinal cord compression (Fig. 2). The mass involved primarily the T8 vertebral body anteriorly, however posterior to the spinal cord, there was a large epidural component from approximately $\mathrm{T} 7-\mathrm{T} 9$. He underwent an emergent $\mathrm{T} 7-\mathrm{T} 9$ open posterior laminectomy and resection of the epidural mass/ tumor (Fig. 1a). The T8 resected epidural mass, bone, and tumor were sent to pathology.

The following morning, he stated that he was feeling much better, and reported increased sensation and movement in the BLEs. The patient was classified as T8 ASIA C-Incomplete Paraplegia. He also presented with neurogenic bladder and 


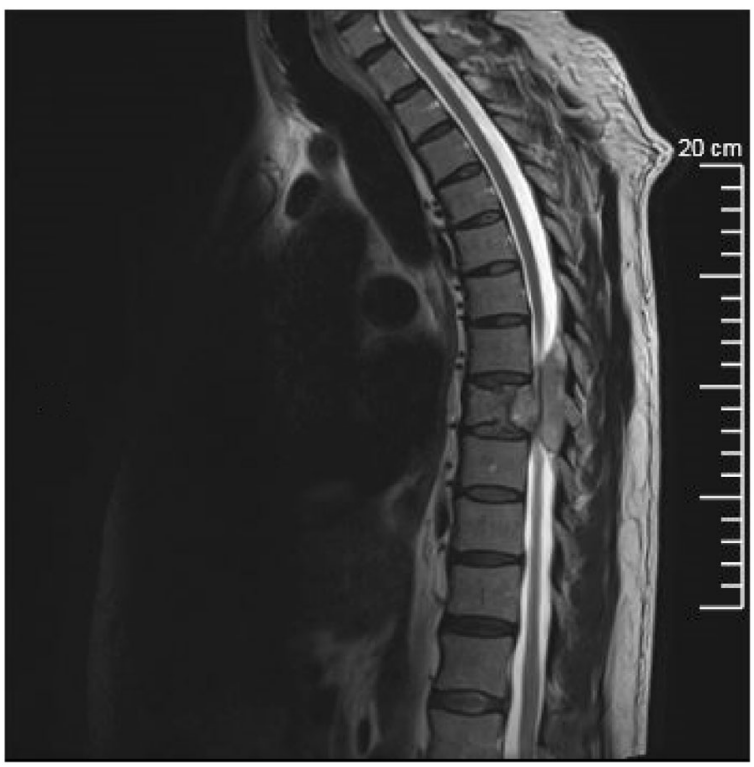

Fig. 2 Spine MRI image revealing a solitary bone plasmacytoma posterior to the spinal cord from approximately $\mathrm{T} 7-\mathrm{T} 9$

bowel, which included a treatment plan consisting of straight catheterization and a reflexic bowl program.

He was admitted to acute inpatient rehabilitation with an intensive therapy regimen (Fig. 1a). At the beginning of acute inpatient rehabilitation, he was using a wheelchair for mobility. MMT revealed improved strength in the BLEs, primarily in the proximal muscles (Fig. 1b). The therapy schedule consisted of: 30-60 min of PT 2 times per day, 5-6 days per week, and 30-60 min of occupational therapy (OT) 2 times per day, 5-6 days per week. In addition, the acute inpatient rehabilitation nursing staff provided nursing cares and SCI education. PT focused on the following: activity tolerance, balance, bed mobility, coordination, gait, stair, transfer, motor coordination, and wheelchair management/propulsion. PT also included strengthening, stretching, range of motion, neuromuscular re-education, and patient/family education. OT included the following: adaptive equipment, activities of daily living, balance, bed mobility, transfer, and energy conservation. OT also focused on endurance within activity guidelines, functional mobility, strengthening and a home exercise program. Over the course of therapy, he continued to gain strength and mobility. By the end of April, he was ambulating short distances with the assistance of a walker.

Based on the pathology results, he was diagnosed with $\mathrm{SP}$ with minimal marrow involvement. The bone marrow biopsy disclosed $<5 \%$ involvement with plasma cell neoplasm. A MRI of the brain was negative for metastatic disease. He had IgG kappa monoclonal gammopathy with a monoclonal spike of 0.6 and elevated kappa free light chains. His urine was positive for free kappa light chains, but no monoclonal spike was detected. He had no other sites of bone involvement on his skeletal survey, and his creatinine, hemoglobin and calcium levels were normal.

Initial post-operative X-rays demonstrated no structural instability. However, several days later, $\mathrm{X}$-ray revealed his T8 vertebra underwent structural collapse associated with thoracic kyphosis, pain, and radiculopathy. The patient had not received adjuvant treatment. At the beginning of May, a T6-T10 open posterior fusion was performed (Fig. 1a). He was readmitted to acute inpatient rehabilitation to continue therapy and was discharged home one week later (Fig. 1a) with improved BLE strength (Fig. 1b).

He began outpatient PT, which totaled 37 sessions over 6 months, with on average 2 sessions per week (Fig. 1a). PT included therapeutic exercise and activities, neuromuscular re-education, gait training, and patient education. Balance was examined in May 2017 using the Berg Balance Scale (BBS). The patient was considered a high fall risk with a BBS score of 20/56 (Fig. 1a).

Over the course of outpatient PT, his strength and mobility continued to improve. By July 2017, he was categorized by the BBS as low fall risk (43/56), he had full BLE strength (Fig. 1b), and he was ambulating independently with a single end cane. By October 2017, his BBS was 55/56 (Fig. 1a) and he was ambulating independently without an assistive device. He was discharged from outpatient physical therapy with a home exercise program. In March 2018, he had a follow up physical therapy appointment, where he displayed retained balance (Fig. 1a) and BLE strength (Fig. 1b).

The oncology course of treatment included radiation with 44 Gy to the T7-T9 vertebral levels, which occurred from 5/ 2017-6/2017. He began a monthly chemotherapy regimen of a $4 \mathrm{mg}$ zoledronic acid infusion in August 2017. Currently, chemotherapy treatments are still ongoing.

\section{Discussion}

SP is an uncommon malignancy caused by abnormal production of monoclonal plasma cells, resulting in a single mass in a localized area. Of all plasma cell neoplasms, only $5 \%$ are estimated to be caused by SP [4]. Diagnostic criteria of SP include the following: (1) solitary lesion of tissue with biopsy evidence of clonal plasma cells, (2) no evidence of clonal plasma cells in bone marrow, (3) normal MRI (aside from primary solitary lesion) of spine and pelvis, and (4) absence of end organ damage [5].

The average age of patients diagnosed with SP is 55-60 years old [3]. The incidence of SP in males is significantly higher than the incidence in females [6]. Additionally, it has been found that the incidence of SP in black individuals is $30 \%$ higher than the incidence in white individuals [6]. Our 
patient, being a 67-year-old African American Male, exemplifies the typical patient demographic associated with SP. Outcomes for SP patients vary significantly by age, but SP patients over the age of 60 are more likely to have less favorable outcomes and progress to multiple myeloma (MM) [6-8]. Specifically, Jawad and colleagues found that age over 60 years was the only factor that correlated with the progression of SP [8].

$\mathrm{SP}$ can be further classified as SBP, SP arising from bone tissue, or Solitary Extramedullary Plasmacytoma (SEP), SP arising from soft tissue [4]. Although the occurrence of SBP is approximately $40 \%$ higher than SEP, SBP has a poorer prognosis [6]. The overall median survival for those diagnosed with SBP averages approximately 10 years $[4,9,10]$. Soutar and colleagues found that more than $75 \%$ of SBP patients progress to MM, while less than $30 \%$ of SEP patients progress [11]. Other studies have found that approximately $50 \%$ of patients diagnosed with SBP progress to MM [10, 12]. On average, SBP progresses to MM within 1.75 to 4 years of diagnosis [10-12]. The described patient is approximately 1.5 years post diagnosis and has not progressed to MM.

Several studies have reported the vertebrae to be the most common site of SBP [4, 10, 13]. Due to lesion compression, back and neck pain are commonly reported initial symptoms of SBP [4], which has been demonstrated by numerous published case reports [14-27]. Of these cases, patients reported pain on average 8 months prior to the diagnosis of SBP. Interestingly, several of these patients were seen by various healthcare providers on multiple occasions during the time leading up to their SBP diagnoses, with some even receiving incorrect diagnoses. In some cases, patients also presented with more severe symptoms, such as progressive paresis or sudden paraplegia [23, 28].

Radiotherapy continues to be the recommended treatment for SBP [29, 30], with local control of patients after moderate doses (40-50 Gy) of radiotherapy [30]. With absence of spinal compression and related symptoms, radiation therapy alone can effectively treat SBP. There were several cases of patients who presented with back pain, were diagnosed with SBP, and were treated with radiotherapy alone [21, 25, 27]. Additionally, some cases of SBP were treated with surgery alone $[16,17,20,22,28]$. However, in cases of spinal compression and neurological symptoms, such as our case, radiotherapy has often been coupled with decompression surgery $[18,19,23,24,26,28]$.

Complete functional and sensory recovery has been reported in cases of SBP following radiotherapy and/or surgery $[14,18,19,26,28]$. Unfortunately, most cases do not report standard SCI classifications or describe the functional recovery in detail. Of the cases that reported functional recovery, it is often unclear whether the patient was diagnosed with SBP or SEP. For example, Tomita and colleagues reported 1 patient with SP improving from Frankel C to D after spondylectomy [31]. In addition, Baba and colleagues reported 8 cases of SP of the spine, where one patient progressed from Frankel grade $\mathrm{B}$ to $\mathrm{E}$ in a period of 3.8 years [32]. Although, it is noteworthy that the patient with this progression was only 49 at the age of SP diagnosis. The researchers also reported that the other 7 patients progressed to Frankel grade D or E, however, two patients were initially classified as Frankel grade $\mathrm{C}$, four at $\mathrm{D}$, and one at $\mathrm{E}$ [32]. Likewise, there have been two reports of full functional recovery after surgery and radiotherapy in individuals with SBP who initially presented with limb weakness and paraparesis $[18,28]$.

This case report is limited by inconsistency in documentation of the gradual return of strength and sensation in the patient. Additionally, the long-term outcomes of the patient are still unknown as the patient is approximately 1.5 years post-diagnosis.

\section{Conclusions}

It has been estimated that in a 1 year period, $6.3-15.4 \%$ of the population presents with a first ever episode of low back pain [33]. Given the complexity of back pain and pain diagnoses in general, there is little data suggesting how many cases of first time presentation of low back pain are the result of SBP. Due to the rarity of the disease, one can assume that many cases of SP are initially misdiagnosed, especially considering the average length of time from initial symptom presentation to diagnosis. We conclude that persistent back pain, especially when coupled with neurologic symptoms, warrants complete MRI spinal imaging to provide proper diagnosis and prompt treatment. Finally, this case suggests that laminectomy and radiotherapy coupled with an intensive rehabilitation regimen may result in a more complete functional recovery in patients with SBP of the spine.

Acknowledgements We would like to thank the patient featured in this case report for generously allowing the publication of personal medical information.

Funding This project was funded by the Research, Education, and Development fund through Regions Hospital, St. Paul, MN.

\section{Compliance with ethical standards}

Conflicts of interest The authors declare no that they have no conflicts of interest.

Publisher's note: Springer Nature remains neutral with regard to jurisdictional claims in published maps and institutional affiliations. 


\section{References}

1. Hoy D, March L, Brooks P, Blyth F, Woolf A, Bain C, et al. The global burden of low back pain: estimates from the Global Burden of Disease 2010 study. Ann Rheum Dis. 2014;73:968-74.

2. National Institute of Neurological Disorders and Stroke. Low Back Pain Fact Sheet. 2017 https://www.ninds.nih.gov/Disorders/ Patient-Caregiver-Education/Fact-Sheets/Low-Back-Pain-FactSheet.

3. Grammatico S, Scalzulli E, Petrucci MT. Solitary plasmacytoma. Mediterr J Hematol Infect Dis. 2017;9:e2017052.

4. Dimopoulos MA, Moulopoulos LA, Maniatis A, Alexanian R. Solitary plasmacytoma of bone and asymptomatic multiple myeloma. Blood. 2000;96:2037-44.

5. Kyle RA, Rajkumar SV. Criteria for diagnosis, staging, risk stratification and response assessment of multiple myeloma. Leukemia. 2009;23:3-9.

6. Dores GM, Landgren O, McGlynn KA, Curtis RE, Linet MS, Devesa SS. Plasmacytoma of bone, extramedullary plasmacytoma, and multiple myeloma: incidence and survival in the United States, 1992-2004. $\mathrm{Br} \mathrm{J}$ Haematol. 2009;144:86-94.

7. Thumallapally N, Meshref A, Mousa M, Terjanian T. Solitary plasmacytoma: population-based analysis of survival trends and effect of various treatment modalities in the USA. BMC Cancer. 2017;17:13.

8. Jawad MU, Scully SP. Skeletal Plasmacytoma: progression of disease and impact of local treatment; an analysis of SEER database. J Hematol Oncol. 2009;2:41.

9. Chak LY, Cox RS, Bostwick DG, Hoppe RT. Solitary plasmacytoma of bone: treatment, progression, and survival. J Clin Oncol. 1987;5:1811-5.

10. Liebross RH, Ha CS, Cox JD, Weber D, Delasalle K, Alexanian R. Solitary bone plasmacytoma: outcome and prognostic factors following radiotherapy. Int $\mathrm{J}$ Radiat Oncol Biol Phys. 1998;41:1063-7.

11. Soutar R, Lucraft H, Jackson G, Reece A, Bird J, Low E, et al. Guidelines on the diagnosis and management of solitary plasmacytoma of bone and solitary extramedullary plasmacytoma. Clin Oncol. 2004;16:405-13.

12. Knobel D, Zouhair A, Tsang RW, Poortmans P, Belkacemi Y, Bolla M, et al. Prognostic factors in solitary plasmacytoma of the bone: a multicenter Rare Cancer Network study. BMC Cancer. 2006;6:118.

13. Guo SQ, Zhang L, Wang YF, Sun BC, Zhang LY, Zhang J, et al. Prognostic factors associated with solitary plasmacytoma. Onco Targets Ther. 2013;6:1659-66.

14. Kulkarni RS, Parikh SK, Anand AS, Panchal HP, Patel AA, Trivedi $\mathrm{P}$, et al. Solitary plasmacytoma of bone involving spine in a 12-year-old boy: report of a rare case and review of literature. J Pediatr Neurosci. 2017;12:67-71.
15. Pashayan R, Cavanaugh WM, Warshel CD, Payne DR. Plasmacytoma of the cervical spine: a case study. J Chiropr Med. 2017;16:170-4.

16. Preston G, Anton T. T2 vertebrectomy with combined anterior and posterior arthrodesis for treatment of a solitary plasmacytoma. Surg Neurol Int. 2017;8:173.

17. Tutar S, Mutlu A, Ozturk E, Ulusoy OL, Enercan M. Solitary plasmacytoma of the thoracic spine. Spine J. 2016;16:e485.

18. Venkatesh R, Tandon V, Patel N, Chhabra HS. Solitary plasmacytoma of L3 vertebral body treated by minimal access surgery: common problem different solution! J Clin Orthop Trauma. 2015;6:259-64.

19. Yang JS, Cho YJ, Kang SH, Choi HJ. Rapid progression of solitary plasmacytoma to multiple myeloma in lumbar vertebra. $\mathrm{J}$ Korean Neurosurg Soc. 2013;54:426-30.

20. Popescu M, Popov V, Popescu G, Dobrea C, Sandu A, Grigorean VT, et al. Spinal involvement with spinal cord compression syndrome in hematological diseases. Rom J Morphol Embryol. 2012;53:1069-72.

21. Rattican D, Kelly DL, Filler KA, Lyon DE. Back pain caused by a solitary plasmacytoma of bone. Clin J Oncol Nurs. 2010;14:149-52.

22. Azarpira N, Vasei M, Rasekhi A. Plasma cell tumors with neurologic symptoms: cytological findings. Diagn Cytopathol. 2012;40:248-51.

23. Terada T. Solitary plasmacytoma of the thoracic vertebra presenting with sudden paraplegia and back pain: a pathologic case report. Pathol Oncol Res: Por. 2011;17:167-9.

24. Cappuccio M, De Iure F, Gasbarrini A, Bandiera S, Boriani S. Solitary plasmacytoma of the spine: a 22 years follow-up case report. Eur Rev Med Pharmacol Sci. 2006;10:251-6.

25. Pai VS. Persistent back pain in adults: Four case reports. J Orthop Surg. 2000;8:65-8.

26. Thorn K, Williams J. Solitary osseus plasmacytoma as a cause of back pain in a young patient. Am J Emerg Med. 1999;17:615-7.

27. Mitchell B, Brukner P. A case of vertebral solitary bone plasmacytoma. Clin J Sport Med. 1998;8:305-7.

28. Takahashi T, Koshu K, Tominaga T, Takahashi A, Yoshimoto T. Solitary plasmacytoma in the thoracic spine. Two case Rep Neurosurg Rev. 1998;21:121-5.

29. McLain RF, Weinstein JN. Solitary plasmacytomas of the spine: a review of 84 cases. J Spinal Disord. 1989;2:69-74.

30. Dagan R, Morris CG, Kirwan J, Mendenhall WM. Solitary plasmacytoma. Am J Clin Oncol. 2009;32:612-7.

31. Tomita K, Kawahara N, Baba H, Tsuchiya H, Fujita T, Toribatake Y. Total en bloc spondylectomy. A new surgical technique for primary malignant vertebral tumors. Spine. 1997;22:324-33.

32. Baba H, Maezawa Y, Furusawa N, Wada M, Kokubo Y, Imura S, et al. Solitary plasmacytoma of the spine associated with neurological complications. Spinal Cord. 1998;36:470-5.

33. Hoy D, Brooks P, Blyth F, Buchbinder R. The epidemiology of low back pain. Best Pract Res Clin Rheumatol. 2010;24:769-81. 